\title{
Placenta Accreta Prenatal Diagnosis: Does MRI add to Sonography?
}

\author{
Sunil Aggarwal ${ }^{1}$ and Somya Aggarwal ${ }^{2 *}$ \\ ${ }^{1}$ Avalon University School of Medicine, Netherlands \\ ${ }^{2}$ Independent Scholar, USA
}

Submission: October 05, 2016; Published: October 24, 2016

*Corresponding author: Somya Aggarwal, Independent Scholar, New York, USA, Tel: 1-717-622-1264; Email: sa8991@rit.edu

\begin{abstract}
The increasing incidence of Placenta accrete has greatly increased the risk of a possible threat to life. The probability of a cesarean section has, thus, increased. Previous cesarean sections with myometrial damage greatly increases the chances of placenta accreta. An emergency approach to its management is necessitated. In this paper we will detail out whether MRI is still required for the diagnosis or prenatal diagnosis though ultrasound is capable of diagnosing placenta accreta.
\end{abstract}

Keywords: Placenta accrete; Placenta increta; Placenta percreta

Abbreviations: MRI: Magnetic Resonance Imaging; PPV: Positive Predictive Value; NPV: Negative Predictive Value

\section{Introduction}

Placenta accreta has the clinical feature of it in- dwelled into the myometrial wall with abnormal implantation and invasion in it. The amount of its in-growth or depth is an important aspect in management, as this pathology is of prime concern in maternal mortality. The diagnosis of placenta accreta or its various descriptive patterns like placenta increta or placenta percreta is usually made by clinically and by the use of Ultrasound and in some cases by MRI. Sometimes, it is just discovered at the time of delivery. Placenta increta refers to invasion of chorionic villi into myometrium whereas placenta percreta goes beyond invasion of myometrium and into serosa and surrounding structures. Massive hemorrhage during delivery due to its non-separation from the uterine wall can lead to other coagulations including disseminate intravascular coagulopathy and may be life threatening.

\section{Risk Factors}

Previous damage to myometrial wall, through prior cesarean section is the most important risk factor for the development of placenta accreta. With the increasing incidence of cesarean section in United States [1], the incidence of placenta accreta is on a rise. There is an increase in the occurrence of placenta accreta due to previous uterine surgeries. This goes on to add many fold to the risk with caesarean and a placenta previa [2].

\section{Pathogenesis of Placenta Accreta}

Primary defects in the myometrial wall are created creating abnormal vascularization after uterine scarring from previous surgery. This causes abnormal decidualization and excessive trophoblastic changes. This is then primarily iatrogenic as there appears to be direct correlation of abnormal placental adherence to prior surgery. Ultrasound studies of cesarean sections scars have elaborated the cause as iatrogenic, although other ill- defined mechanisms may exist [3].

\section{Transvaginal and Trans Abdominal Ultrasound}

Transvaginal and Trans abdominal ultrasonographies are two modes of ultrasounds readily available for scanning and should complement each other and used when required. Placenta previa patients may be scanned by transvaginal approach for a complete diagnostic picture and would show an irregular lacunae or vascular spaces in the placenta, instead of the normal hypo echoic interface between placenta and the urinary bladder [4]. The increase in number of such placental lacunae is thought to be one of the most diagnostic and informative sign at 15-20 weeks of gestation [5], making placenta have a "moth-eaten" or "Swiss cheese" diagnostic appearance. 


\section{Use of Ultra Sonography versus MRI in Placenta accreta}

Usually, ultrasonography is sufficient to predict and let a physician know of impending or present placenta accreta, with a high degree of confidence. Introduction of color imaging in the form of Doppler or 3-D imaging does not further enhance the diagnostic value of grey scale imaging [6].

Routine antenatal sonography would include a possible diagnosis of placenta previa, including accrete, but chorionic villus pathology is the final diagnostic marker. When we compare the statistics of grey scale ultrasound and MRI for placenta accreta, we see that it has a sensitivity of $77 \%$ to $87 \%$, specificity of $96 \%$ to $98 \%$, a positive predictive value (PPV) of $65 \%$ to $93 \%$, and a negative predictive value (NPV) of $98 \%[4,5]$. MRI, on the other hand, has a sensitivity of $80 \%$ to $85 \%$, specificity of $65 \%$ to $100 \%$ [4]. This leads us to the firm opinion of ultrasound to be used as the basic investigation modality for the diagnosis of placenta accreta [5]. MRI adds no further clarity to the diagnosis if used after an ultrasound scan for placenta accrete [6] and may result in both false-positive and false-negative diagnoses for placenta accrete [6].

\section{Conclusion}

It cannot be over emphasized here that the timely diagnosis of placenta accreta helps minimize surgical complications. A detailed prior cesarean section surgeries information coupled with timely diagnosis can lead in minimizing blood loss, and better surgical plans. Since real time ultra sonography is available at nearly all the imaging centers, less expensive and non- invasive, with a good diagnostic value, it should be the first and foremost line of investigation in radiological investigations as the modality of choice for placenta accreta. Transvaginal ultrasonography would be the best indicator of placenta accreta and rarely would MRI be required to top it up. MRI should be reserved if ultrasound is non- diagnostic. Will it add to ultrasound in further diagnosis of placenta accreta is not to be seen in various studies and should be used when absolutely required to [7].

\section{References}

1. Blanchette $H(2011)$ The rising cesarean delivery rate in America: what are the consequences? Obstet Gynecol 118(3): 687-690.

2. Fitzpatrick KE, Sellers S, Spark P, Kurinczuk JJ, Brocklehurst P, et al. (2012) Incidence and Risk Factors for Placenta Accreta/Increta/ Percreta in the UK: A National Case-Control Study. PLoS ONE 7(12): e52893.

3. Basic E, Basic-Cetkovic V, Kozaric H, Rama A (2012) Ultrasound Evaluation of Uterine scar after cesarean section. Acta Informatica Medica 20(3): 149-153.

4. Comstock CH, Love JJ, Bronsteen RA, Lee W, Vettraino IM, et al. (2004) Sonographic detection of placenta accreta in the second and third trimesters of pregnancy. Am J Obstet Gynecol 190(4): 1135-1140.

5. Warshak CR, Eskander R, Hull AD, Scioscia AL, Mattrey RF, et al. (2006) Accuracy of ultrasonography and magnetic resonance imaging in the diagnosis of placenta accreta. Obstet Gynecol 108(3 Pt 1): 573-578.

6. Chou MM, Ho ES, Lee YH (2000) Prenatal diagnosis of placenta previa accreta by transabdominal color Doppler ultrasound. Ultrasound Obstet Gynecol 15(1): 28-35.

7. Kanal E, Barkovich AJ, Bell C, Borgstede JP, Bradley WG, et al. (2007) ACR guidance document for safe MR practices: 2007. ACR Blue Ribbon Panel on MR Safety. AJR Am J Roentgenol 188(6): 1447-1474. 